\title{
Acute kidney injury
}

\author{
Authors: Alistair Connell ${ }^{\mathrm{A}}$ and Chris Laing ${ }^{\mathrm{B}}$
}

Acute kidney injury (AKI) - an abrupt deterioration in renal function - causes a rise in serum creatinine $(\mathrm{SCr})$ or fall in urine output. It is common, occurring in up to $20 \%$ of hospital admissions. Importantly, even small rises in $\mathrm{SCr}$ are associated with increased risk of death and longer hospital stays. A 2009 National Confidential Enquiry into Patient Outcome and Death report found that a proportion of AKI in secondary care was avoidable. In addition, management of established AKI was 'good' less than half the time. In practice, AKI represents a heterogeneous group of conditions, encompassing impairments in both kidney structure and function. Delivering disease-specific treatment early in the course of AKI may improve outcomes. The provision of best-practice care for all will rely on a better understanding of risk, and frameworks of care that can be applied across a diverse patient group.

\section{Introduction - definitions and importance}

Acute kidney injury (AKI) - an abrupt deterioration in renal function- causes a rise in serum creatinine (SCr) and/or fall in urine output. The magnitude of these changes is used to stratify the severity of AKI, as defined in an internationally agreed system of classification designed for clinical practice and research (KDIGO). ${ }^{1}$ Using this classification, AKI is common, occurring in up to $20 \%$ of hospital admissions. ${ }^{2}$ Importantly, even small rises in SCr are independently associated with an increased risk of death. ${ }^{2}$ Some 40,000 excess deaths are attributed to AKI each year in England alone; ${ }^{3}$ a mortality burden greater than that attributable to MRSA and venous thromboembolism combined. ${ }^{4}$ Morbidity is also high in AKI; it is associated with an increased length of stay, ${ }^{3}$ and the development of chronic kidney disease. ${ }^{5}$ This is expensive; one recent economic analysis estimated the associated healthcare costs for England alone to be over $£ 1$ billion per annum; a yearly cost that exceeds treatment for some major cancers. ${ }^{3}$

In practice, AKI represents a heterogeneous group of conditions, encompassing impairments in kidney structure and function. Of those diagnosed with AKI in hospital, roughly half will have effective hypovolaemia or sepsis. ${ }^{6}$ The remaining cases are caused by a mixture of obstructive uropathy, nephrotoxins

Authors: ${ }^{\text {A }}$ linical fellow, Institute of Human Health and Performance, UCL, London, UK; ${ }^{B}$ consultant nephrologist, Department of Nephrology, Royal Free London NHS Foundation Trust, London, UK and a variety of parenchymal renal diseases. The London AKI Network has produced comprehensive guidelines and care pathways, designed to assist clinicians in routine management. Their summary of the main causes of AKI is shown in Box 1 .

\section{Understanding risk in AKI}

A 2009 National Confidential Enquiry into Patient Outcome and Death (NCEPOD) report found that the care of patients with established AKI was 'good' less than half the time. ${ }^{7}$ The potential for the reduction of patient morbidity, mortality and healthcare costs in AKI are thus clear. Alongside sepsis, the NHS

Box 1. The primary causes of AKI. Reproduced with permission from the London AKI Network.

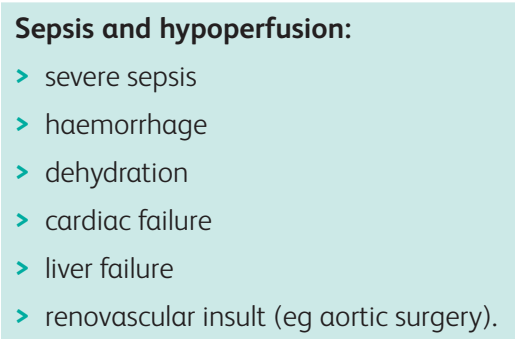

Toxicity:

$>$ nephrotoxic drugs

iodinated radiological contrast.

\section{Obstruction:}

> bladder outflow

$>$ stones

$>$ tumour

$>$ surgical ligation of ureters

$>$ extrinsic compression (eg lymph nodes)

$>$ retroperitoneal fibrosis.

Parenchymal kidney disease:

glomerulonephritis

$>$ tubulointersitial nephritis

$>$ rhabdomyolysis

> haemolytic uraemic syndrome

myeloma kidney

malignant hypertension. 


\section{AKI Care Bundle}

Fig 1. An AKI care bundle. Reproduced with permission from the London AKI Network. ACE = angiotensin-converting-enzyme; $\mathrm{AKI}=$ acute kidney injury; $\mathrm{ARB}=$ angiotensin-receptor blocker; Bili = bilirubin; $C K=$ creatine kinase; $\mathrm{CRP}=\mathrm{C}$-reactive protein; HUS = hemolytic-uremic syndrome; $\mathrm{LDH}=$ lactate dehydrogenase; NSAIDs = non-steroidal anti-inflammatory drugs; $P C R=$ protein-creatinine ratio; retics $=$ reticulocytes; TTP = thrombotic thrombocytopenic purpura.
Institute in all patients with a $1.5 \mathrm{X}$ rise in creatinine or oliguria

$(<0.5 \mathrm{~mL} / \mathrm{kg} / \mathrm{h}$ ) for $>6 \mathrm{~h}$

This is a medical emergency

Full set of physiological observations; assess for signs of shock/hypoperfusion; if MEWS

triggering give oxygen, begin resuscitation and contact critical care outreach team

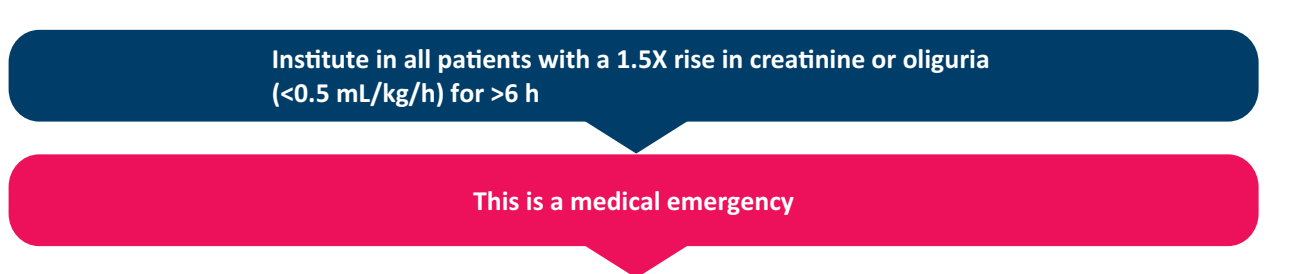

Fluid therapy in AKI

$>$ Assess heart rate, blood pressure, jugular venous pressure, capillary refill (should be $<3 \mathrm{sec}$ ), conscious level.

$>$ If hypovolaemic give bolus fluids (eg 250-500 mL) until volume replete with regular review of response.

$>$ Middle grade review if $>2 L$ filling in oliguria.

$>$ If the patient is euvolaemic give maintenenance fluids (estimated output plus $500 \mathrm{~mL}$ ) and set daily fluid target.

Monitoring in AK

$>$ Do arterial blood gas and lactate if venous bicarbonate is low or evidence of severe sepsis or hypoperfusion.

$>$ Consider insertion of urinary catheter and measurement of hourly urine volumes.

$>$ Measure urea, creatinine, bone, other electrolytes and venous bicarbonate at least daily while creatinine rising.

$>$ Measure daily weights, keep a fluid chart and perform a minimum of 4 hourly observations.

$>$ Perform regular fluid assessments and check for signs of uraemia.

\section{Investigation of AKI}

$>$ Investigate the cause of all AKI unless multi-organ failure or obvious precipitant

$>$ Urine dipstick: if proteinuria is present perform urgent spot urine PCR.

USS should be performed within $24 \mathrm{~h}$ unless AKI cause is obvious or AKI is recovering

or within 6 hours if obstruction with infection (pyonephrosis) is suspected.

$>$ Check liver function (hepatorenal), CRP and CK (rhabdomyolysis): if platelets low do blood

film/LDH/Bili/retics (HUS/TTP). If PCR high, consider urgent Bence Jones protein and serum free light chains.

\section{Supportive AKI care}

$>$ Treat sepsis - in severe sepsis intravenous antibiotics should be administered within 1 hour of recognition.

$>$ Stop NSAID/ACE/ARB/metformin/K-sparing diuretics and review all drug dosages.

$>$ Give proton pump inhibitor and perform dietetic assessment.

$>$ Stop anti-hypertensives if relative hypotension. If hypovolaemic consider stopping diuretics.

Avoid radiological contrast if possible. If given follow prophylaxis protocol.

Causes think 'STOP AKI'

Sepsis and hypoperfusion, toxicity (drugs/contrast), obstruction, parenchymal kidney disease (acute GN)
Five year forward view identified improving AKI care as an area that will maximally reduce avoidable mortality. ${ }^{8}$ Improving outcomes at scale will depend on better understanding of risk, and the consistent delivery of best-practice care.

The 2009 NCEPOD report also highlighted that up to $20 \%$ of AKI in secondary care was avoidable. The chance of developing AKI following exposure to an insult depends on a host of individual factors. These relate to both underlying susceptibilities (eg existing chronic kidney disease or diabetes) and exposures in hospital (eg sepsis or major surgery). ${ }^{9}$ Care should be taken to ensure that these exposures are minimised for patients at risk; this will include ensuring volume status is optimised and the avoidance of nephrotoxins. Monitoring of serum creatinine and urine output is also important for early recognition of AKI; but it should also be borne in mind that rises in SCr will lag behind any drops in in glomerular filtration rate following an insult to the kidneys by several days.

\section{An organised approach to patient management}

While the relationship between AKI, critical illness and comorbidities is complex, there is broad recognition that a 
strategy of early intervention may avoid significant parenchymal injury in patients with multi-system illness. ${ }^{10,11}$ Early review by a nephrology team may help ameliorate $\mathrm{AKI},{ }^{12}$ but the numbers of patients developing AKI and the centralisation of services make this impossible for all. As a result, local and regional care protocols exist for monitoring, investigation and supportive care (see Fig 1 for an example of such a pathway). ${ }^{13}$

\section{Managing complications and tertiary referral}

Patients with established AKI must also be monitored for complications; some may need to be cared for in higher acuity areas of care. Care protocols can guide immediate management and ensure patient safety (see Fig 2).

In addition, for any AKI patient with one of the above complications, local critical care services should be contacted for patients with AKI3 (serum creatinine $>3 \mathrm{X}$ baseline OR $>354 \mu \mathrm{mol} / \mathrm{L}$ OR initiation of renal replacement therapy), or those in whom imminent recovery is unlikely. Urgent referral to local urological services is warranted for those with obstruction on ultrasound. In the first instance, referrals to local nephrology services should be reserved for patients with AKI and the following:

$>$ myeloma

$>$ suspected autoimmune disease or glomerulonephritis (positive autoimmune screen; blood or protein on urine dip)

> suspected hemolytic-uremic syndrome and thrombotic (history of diarrhoea or rash)

$>$ thrombocytopenic purpura

$>$ suspected poisoning.

\section{Future frameworks for care}

Recent local and national initiatives have focused on early recognition and timely intervention, leading to the adoption

\section{AKI Complications}

Hyperkalaemia, acidosis, pulmonary oedema, reduced conscious level

Begin medical therapy and get help

Local critical care team and local nephrology team (if onsite)

Hyperkalaemia

$>$ Medical therapy of hyperkalaemia is a transient measure pending imminent recovery in renal function or transfer to kidney unit or critical care for renal replacement therapy.

$>$ If ECG changes give calcium gluconate $10 \mathrm{~mL} 10 \%$.

$>$ If bicarbonate $<22 \mathrm{mmol} / \mathrm{L}$ and no fluid overload give $500 \mathrm{~mL} 1.26 \%$ sodium bicarbonate over $1 \mathrm{~h}$.

$>\mathrm{K}>6.5 \mathrm{mmol} / \mathrm{L}$ or ECG changes give insulin $10 \mathrm{IU}$ in $50 \mathrm{~mL}$ of $50 \%$ dextrose over $15 \mathrm{~min}$ and salbutamol $10 \mathrm{mg}$ nebulised (caution with salbutamol in tachycardia or ischaemic heart disease). Insulin/dextrose and salbutamol reduce ECF potassium for $<4 \mathrm{~h}$ only.

Acidosis

$>$ Medical therapy of acidosis with bicarbonate should be reserved fo emergency management of hyperkalaemia (as above) pending specialist help.

$>\mathrm{pH}<7.15$ requires immediate critical care referral.

Pulmonary oedema

$>$ Sit the patient up and give oxygen (60-100\% unless contraindicated).

$>$ If haemodynamically stable give furosemide $80 \mathrm{mg}$ iv. Consider repeat bolus and infusion at $10 \mathrm{mg} / \mathrm{h}$.

$>$ If haemodynamically stable commence GTN $1-10 \mathrm{mg} / \mathrm{h}$ titrating dose.

Reduced conscious level

Manage uraemic coma as per all reduced consciousness (airway management) pending critical care transfer and emergency renal replacement therapy.

These are holding measures prior to specialist help from critical care or nephrology services.
Fig 2. Managing complications in AKI. Reproduced with permission from the London AKI Network. AKI = acute kidney injury; ECG = echocardiogram; $\mathrm{ECF}=$ extracellular fluid; GTN = glyceryl trinitrate. 


\section{Key points}

Acute kidney injury (AKI) reflects a heterogeneous group of structural and functional diseases of the kidney.

It is common, and associated with significant morbidity and mortality.

A proportion of AKI is predictable, preventable or recognised late.

Risk relates to both acute insults and comorbid disease.

Care bundles can ensure timely diagnosis, appropriate supportive care, targeted therapy and referral.

KEYWORDS: Nephrology, acute kidney injury

of real-time automated diagnostic algorithms. ${ }^{14} \mathrm{NHS}$ England have produced one such algorithm, mandating that it be incorporated in some way into all hospital laboratory information management systems. Alerts for patients with AKI are to be displayed in patient management systems and reported centrally. ${ }^{15}$ However, trials of automated clinical decision support tools have had mixed results. A recent randomised controlled trial testing the efficacy of simple electronic e-alerting for AKI resulted in no significant differences in patient peak creatinine, requirement for dialysis or risk of death. ${ }^{16}$ As discussed above, patients with AKI form a heterogeneous group, and therapy is not standardised; a new process may be needed to guide the nature and scale of clinical response in the right timeframe. It has already been demonstrated that existing patient data can be used to reduce false positive alerts (eg patients with chronic kidney disease or on renal replacement therapy). ${ }^{17}$ As computers become more powerful and patient-specific data are coded with greater granularity, the potential for more advanced risk prediction using artificial intelligence is huge.

Current approaches in the diagnosis of AKI are also limited by the regularity with which SCr or urine output are measured. In the future, strategies may focus on fostering early detection and more targeted therapy. Numerous novel biomarkers reflecting function or structural damage to the kidney have been investigated (neutrophil gelatinase-associated lipocalin, kidney injury molecule 1). Although there is hope that they may one day facilitate earlier diagnosis or differentiate separate causal pathways, current approaches lack sensitivity and may be less effective at the level of the individual patient. Crucially, they have yet to consistently show value in clinical decision making above and beyond existing SCr and urine output monitoring. Future efforts may focus on their use alongside existing biochemical and clinical information. ${ }^{18}$

\section{References}

1 KDIGO. KDIGO Clinical practice guideline for acute kidney injury. Kidney Int Suppl 2012;2:1-138.

2 Wang HE, Muntner P, Chertow GM et al. Acute kidney injury and mortality in hospitalized patients. Am J Nephrol 2012;35:349-55.

3 Kerr M; Bedford M; Matthews B et al. The economic impact of acute kidney injury in England. Nephrol Dial Transplant 2014;29:1362-8.

4 Fluck R. Acute kidney injury: common, harmful, treatable. London: NHS England. Available online at www.england.nhs.uk/wpcontent/uploads/2014/03/bg-present-keep-kidneys.pdf [Accessed 5 October 2015].

5 Coca SG, Singanamala S, Parikh CR. Chronic kidney disease after acute kidney injury: a systematic review and meta-analysis. Kidney Int 2012;81:442-8.

6 Chawla LS. Disentanglement of the acute kidney injury syndrome. Curr Opin Crit Care 2012;82:516-24.

7 National Confidential Enquiry into Patient Outcome and Death. Acute kidney injury: adding insult to injury. London: NCEPOD, 2009. Available online at www.ncepod.org.uk/2009aki.htm [Accessed 5 October 2015].

8 NHS England. The forward view into action: planning for 2015/16. London: NHS England. London: NHS England, 2014. Available online at www.england.nhs.uk/wp-content/uploads/2014/12/ forward-view-plning.pdf [Accessed 5 October 2015].

9 Kellum JA, Lameire N; KDIGO AKI Guideline Work Group. Diagnosis, evaluation, and management of acute kidney injury: a KDIGO summary (Part 1). Crit Care 2013;17:204.

10 Ronco C, Kellum JA, Bellomo R et al. Potential Interventions in SepsisRelated Acute Kidney Injury. Clin J Am Soc Nephrol 2008;3:531-44.

11 Schrier RW. Fluid administration in critically ill patients with acute kidney injury. Clin J Am Soc Nephrol 2010;5:733-9.

12 Balasubramanian G, Al-Aly Z, Moiz A et al. Early nephrologist involvement in hospital-acquired acute kidney injury: a pilot study. Am J Kidney Dis 2011;57:228-34.

13 London Acute Kidney Injery Network. Guidelines and pathways. London: London AKI Network, 2015. Available online at www. londonaki.net/clinical/guidelines-pathways.html [Accessed 5 October 2015].

14 Selby NM, Crowley L, Fluck RJ et al. Use of electronic results reporting to diagnose and monitor AKI in hospitalized patients. Clin J Am Soc Nephrol 2012;7:533-40.

15 NHS England. NHS Patient Safety Alert: NHS/PSA/D/2014/010. London: NHS England, 2014. Available online at www.england.nhs. uk/wp-content/uploads/2014/06/psa-aki.pdf [Accessed 5 October 2015].

16 Wilson FP, Shashaty M, Testani J et al. Automated, electronic alerts for acute kidney injury: a single-blind, parallel-group, randomised controlled trial. Lancet 2015;385: 1966-74.

17 Celi LAG, Tang RJ, Villarroel MC et al. A clinical database-driven approach to decision support: predicting mortality among patients with acute kidney injury. J Healthc Eng 2011;2:97-110.

18 Lameire NH, Vanholder RC, Van Biesen WA. How to use biomarkers efficiently in acute kidney injury. Kidney Int 2011;79:1047-50.

Address for correspondence: Dr A Connell, 170 Tottenham

Court Road, London W1T 7HA, UK.

Email: alistair.connell@ucl.ac.uk 